\title{
The higher spin generalization of the 6-vertex model with domain wall boundary conditions and Macdonald polynomials
}

\author{
Tiago Fonseca - Ferenc Balogh
}

Received: 18 October 2012 / Accepted: 18 August 2014 / Published online: 9 September 2014

(C) Springer Science+Business Media New York 2014

\begin{abstract}
The determinantal form of the partition function of the 6-vertex model with domain wall boundary conditions was given by Izergin. It is known that for a special value of the crossing parameter the partition function reduces to a Schur polynomial. Caradoc, Foda and Kitanine computed the partition function of the higher spin generalization of the 6-vertex model. In the present work, it is shown that for a special value of the crossing parameter, referred to as the combinatorial point, the partition function reduces to a Macdonald polynomial.
\end{abstract}

Keywords Quantum integrable systems · Combinatorics · Mathematical physics · Symmetric polynomials $\cdot 6$ Vertex model

\section{Introduction}

The 6-vertex model with domain wall boundary conditions was introduced by Korepin in [11], where the partition function was shown to satisfy certain recursion relations.

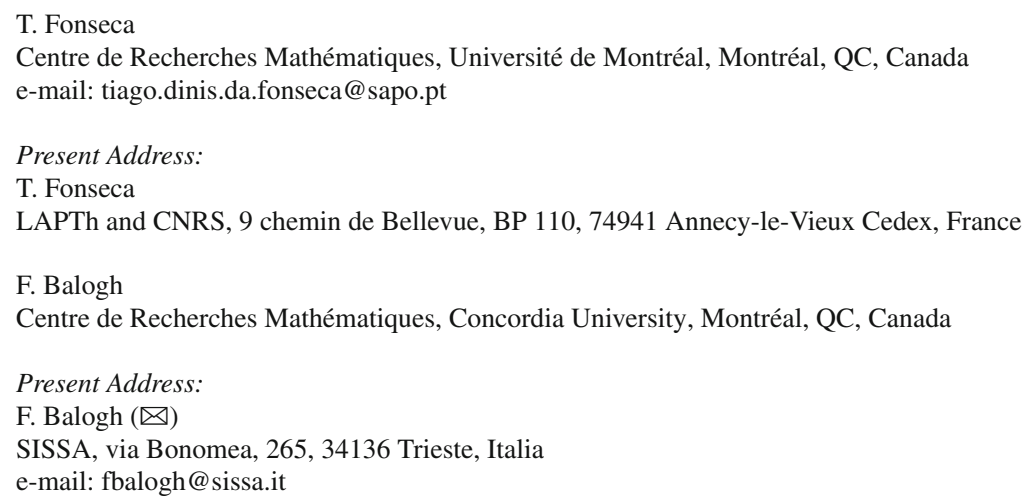


In [10], Izergin solved Korepin's recursion relations, expressing the partition function in a determinantal form.

An interesting feature of the 6-vertex model is that it has multiple combinatorial interpretations since its configurations are in bijection with several combinatorial objects, such as alternating sign matrices, fully packed loops and states of a square ice model [2,4,13,14,17,18]. For example, Kuperberg [13] used the 6-vertex model to compute the number of alternating sign matrices.

The 6-vertex model is an integrable model, meaning that it possesses an $R$-matrix which satisfies the Yang-Baxter equation. It is this algebraic structure that allows the partition function to be calculated explicitly.

To each edge of the 6-vertex model, a $1 / 2$ spin is associated, since the corresponding $R$-matrix is intimately connected with the representation theory of the algebra $\mathfrak{s l}_{2}$. It is natural to search for generalizations of this model, where the representation of the underlying algebra or even the algebra itself is replaced (for example, by $\mathfrak{s l}_{r}$ ). The idea is to construct an $R$-matrix for the model that satisfies the Yang-Baxter equation (see, e.g. [20]).

If we restrict ourselves to studying general irreducible representations of $\mathfrak{s l}_{2}$, there is a systematic way of constructing the $R$-matrix, referred to as fusion [12,21]. The idea comes from the simple fact that in the representation theory of $\mathfrak{s l}_{2}$ we can build the spin $\ell / 2$ representation through the fusion of $\ell$ spin $1 / 2$ representations. This was achieved, for the 6-vertex model with domain wall boundary conditions, in the work of Caradoc, Foda and Kitanine [3], which serves as the basis of our paper.

The partition function of the 6-vertex model, as defined in Sect. 2, is a multivariate polynomial which is symmetric in two separate sets of variables (known as spectral parameters). It also depends on an extra parameter, normally denoted by $q$, and is referred to as the crossing parameter.

If we set $q=\exp (2 \pi i / 3)$, known as the combinatorial point, the partition function becomes symmetric in the two sets of variables as a whole, and it simplifies to a Schur polynomial corresponding to a staircase partition (see [19,23]).

The main goal of the present work is to prove an analogous result for the higher spin generalization of the six-vertex model with domain wall boundary conditions: by setting $q=\exp (2 \pi i /(2 \ell+1))$, the partition function reduces to a Macdonald polynomial corresponding to a staircase partition. As a consequence, the partition function is a symmetric polynomial in the full set of spectral parameters.

\subsection{Outline of the paper}

The first two sections are introductory: Sect. 2 gives the definition of the 6-vertex model with domain wall boundary conditions and the explicit determinantal form of the partition function. In Sect. 3, the higher spin generalization of the 6-vertex model and the analogous representation of its partition function are presented.

The following two sections contain the original results of the paper: Sect. 4 presents an alternative representation of the partition function in terms of determinants of scalar products of rational functions, and this reformulation is used to prove some basic but important properties of the partition function, valid for all values of $q$. 
In Sect. 5, we prove that the partition function satisfies the wheel condition when $q=\exp (2 \pi i /(2 \ell+1))$, and, using the results of [6], we show that there is a welldefined unique Macdonald polynomial satisfying the same vanishing constraints. It is shown that, up to a multiplicative constant, there is a unique polynomial with the prescribed degrees and symmetries that satisfies the wheel condition, and therefore, the partition function and the above mentioned uniquely fixed Macdonald polynomial coincide, up to an explicit multiplicative constant. The proof of the uniqueness lemma and the calculation of the proportionality constant are left to the appendices.

\section{Review of the 6-vertex model}

In this section, we give a brief description of the 6-vertex model, on a square grid, with domain wall boundary conditions, following the construction presented in [8].

\subsection{Definition of the model}

Take a square grid of size $n \times n$, in which each edge is given an orientation (an arrow), such that at each vertex there are two incoming and two outgoing arrows, which gives six possibilities. Alternatively, the arrows can be represented by signs according to the rule that arrows pointing right or upward correspond to plus signs and arrows pointing downward or left correspond to minus signs. Impose the domain wall boundary conditions prescribing that the arrows at the top and bottom boundaries are outgoing, and the ones at the left and right boundaries are incoming. See, e.g. Fig. 1.

To each vertex configuration we assign a weight

$$
w(x, y)=\left\{\begin{array}{l}
a(x, y)=q x-q^{-1} y \\
b(x, y)=x-y \\
c(x, y)=\left(q-q^{-1}\right) \sqrt{x y}
\end{array}\right.
$$
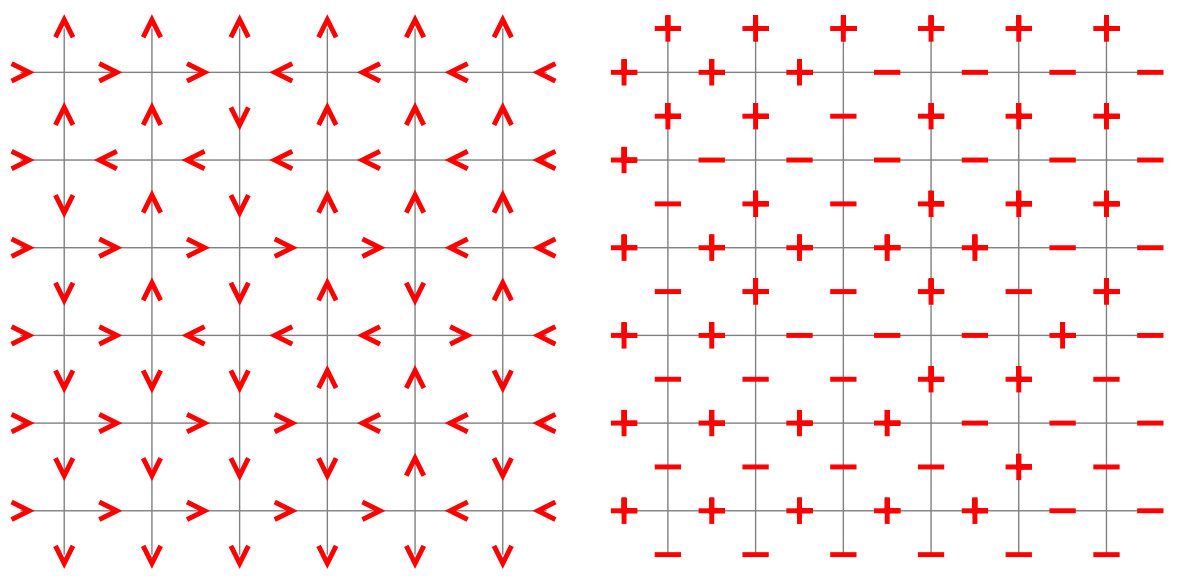

Fig. 1 A $6 \times 6$ configuration of the 6-vertex model in terms of arrows (left) or signs (right) 


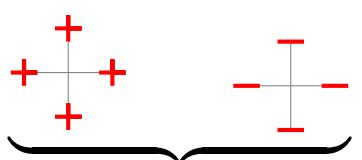

$a(x, y)$
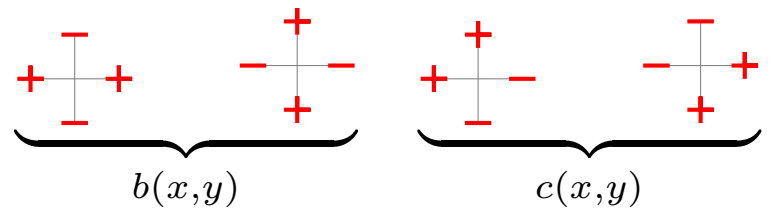

Fig. 2 Weights of vertex configurations

according to Fig. 2. The parameter $q$ is called the crossing parameter of the model, while the parameters $x$ and $y$, called spectral parameters, depend on the row and the column of the vertex, respectively. Let $\boldsymbol{x}=\left\{x_{1}, \ldots, x_{n}\right\}$ and $\boldsymbol{y}=\left\{y_{1}, \ldots, y_{n}\right\}$ be the horizontal and vertical spectral parameters, respectively.

The weight of a configuration is defined by the product of the weights of the vertices. The partition function is defined as the sum of the weights over all possible configurations:

$$
Z_{n}(\boldsymbol{x}, \boldsymbol{y}):=\sum_{\text {configurations }} \prod_{i, j=1}^{n} w_{i j}\left(x_{i}, q y_{j}\right)
$$

where we have chosen to rescale the vertical spectral parameters by a factor of $q$. The partition function is renormalized in the following way:

$$
\mathcal{Z}_{n}(\boldsymbol{x}, \boldsymbol{y})=(-1)^{\left(\begin{array}{c}
n \\
2
\end{array}\right)} q^{-n^{2} / 2}\left(q-q^{-1}\right)^{-n}\left(\prod_{i=1}^{n} x_{i}^{-1 / 2} y_{i}^{-1 / 2}\right) Z_{n}(\boldsymbol{x}, \boldsymbol{y}) .
$$

The function $\mathcal{Z}_{n}(\boldsymbol{x}, \boldsymbol{y})$ is a homogeneous polynomial of total degree $n(n-1)$ and of degree $n-1$ in each variable $x_{i}$ or $y_{i}$.

\subsection{Integrability}

Let $V$ be the standard representation of $\mathfrak{s l}_{2}$ spanned by the eigenvectors $|+\rangle$ and $|-\rangle$ of $S^{z}$. The key ingredient to the exact solvability of the 6-vertex model is the $R$-matrix

$$
R(x, y):=\left(\begin{array}{cccc}
a & 0 & 0 & 0 \\
0 & b & c & 0 \\
0 & c & b & 0 \\
0 & 0 & 0 & a
\end{array}\right)
$$

representing an endomorphism

$$
\begin{aligned}
& R: V \otimes V \rightarrow V \otimes V \\
& \left|\epsilon_{1}\right\rangle \otimes\left|\epsilon_{2}\right\rangle \mapsto R_{\epsilon_{1} \epsilon_{2}}^{\epsilon_{3} \epsilon_{4}}\left|\epsilon_{3}\right\rangle \otimes\left|\epsilon_{4}\right\rangle,
\end{aligned}
$$

where $\epsilon_{i} \in\{+,-\}$. In what follows, we consider the vector space $\bigotimes_{k=1}^{n} V_{k}$, where each $V_{k}$ is a labelled copy of $V$. We use the abbreviated notation 


$$
\left|\epsilon_{1} \epsilon_{2} \cdots \epsilon_{n}\right\rangle:=\left|\epsilon_{1}\right\rangle \otimes\left|\epsilon_{2}\right\rangle \otimes \cdots \otimes\left|\epsilon_{n}\right\rangle, \quad \epsilon_{i}= \pm \quad(i=1,2, \ldots, n)
$$

for a canonical basis in the tensor product representation. The matrix $R_{i j}$ stands for the map that acts as $R$ on $V_{i} \otimes V_{j}$ and as the identity elsewhere.

The action of the matrix $R$ can be interpreted as completing an allowed sign configuration at a vertex with prescribed left and bottom signs, as shown below:

$$
\left.\epsilon_{1}\right|_{\epsilon_{2}} ^{\epsilon_{4}} \epsilon_{3}
$$

The $R$-matrix satisfies the Yang-Baxter equation:

$$
R_{23}\left(y_{2}, y_{3}\right) R_{13}\left(y_{1}, y_{3}\right) R_{12}\left(y_{1}, y_{2}\right)=R_{12}\left(y_{1}, y_{2}\right) R_{13}\left(y_{1}, y_{3}\right) R_{23}\left(y_{2}, y_{3}\right) \text {, }
$$

and the inversion equation:

$$
R_{21}(y, x) R_{12}(x, y)=\left(q y-q^{-1} x\right)\left(q x-q^{-1} y\right) \text { Id } .
$$

The transfer matrix of the model is defined as

$$
T(x, y)={ }_{0}\left\langle-\left|R_{0 n}\left(x, q y_{n}\right) \ldots R_{02}\left(x, q y_{2}\right) R_{01}\left(x, q y_{1}\right)\right|+\right\rangle_{0},
$$

where the matrix $R_{0 i}$ acts on the tensor product of the $i^{\text {th }}$ space and the so-called auxiliary space $V_{0}$. In terms of the transfer matrix, the partition function is given by

$$
Z_{n}(\boldsymbol{x}, \boldsymbol{y})=\left\langle++\cdots+\left|T\left(x_{1}, \boldsymbol{y}\right) T\left(x_{2}, \boldsymbol{y}\right) \ldots T\left(x_{n}, \boldsymbol{y}\right)\right|--\cdots-\right\rangle .
$$

Using the Yang-Baxter equation, the renormalized partition function $\mathcal{Z}_{n}(\boldsymbol{x}, \boldsymbol{y})$, defined in Eq. (3), is shown to be the so-called Izergin-Korepin determinant $[10,11]$

$$
\mathcal{Z}_{n}(\boldsymbol{x}, \boldsymbol{y})=\frac{\prod_{i, j}\left(x_{i}-q y_{j}\right)\left(x_{i}-q^{-1} y_{j}\right)}{\Delta(\boldsymbol{x}) \Delta(\boldsymbol{y})} \operatorname{det}_{1 \leq i, j \leq n}\left(\frac{1}{\left(x_{i}-q y_{j}\right)\left(x_{i}-q^{-1} y_{j}\right)}\right)
$$

where $\Delta(\boldsymbol{x})=\prod_{1 \leq i<j \leq n}\left(x_{i}-x_{j}\right)$.

\subsection{Combinatorial point}

When $q=\exp (2 \pi i / 3)$, the Izergin-Korepin determinant (11) dramatically simplifies and becomes a Schur polynomial $[19,23]$ :

$$
\mathcal{Z}_{n}(\boldsymbol{x}, \boldsymbol{y})=s_{\delta_{n}}(\boldsymbol{x}, \boldsymbol{y})
$$

where $\delta_{n}=(n-1, n-1, n-2, \ldots, 2,1,1,0,0)$. It follows that the partition function $\mathcal{Z}_{n}(\boldsymbol{x}, \boldsymbol{y})$, at the combinatorial point, is a fully symmetric polynomial in the $2 n$ variables $\{\boldsymbol{x}, \boldsymbol{y}\}$. 


\section{Fusion and the higher spin generalization of the 6-vertex model}

We introduce the higher spin generalization of the 6-vertex model considered in this paper. The corresponding $R$-matrix is constructed using fusion techniques for the representations of $\mathfrak{s l}_{2}$, as briefly explained below (see [21]).

\subsection{Fusion and the generalized $R$-matrix}

The representation $\operatorname{Sym}^{\ell} V$ is the irreducible component of $V^{\otimes \ell}$ spanned by the vectors

$$
|\ell ; \ell-m\rangle:=\frac{1}{m !}\left(S^{-}\right)^{m}|\underbrace{++\ldots+}_{\ell}\rangle \quad m=0,1, \ldots, \ell
$$

(see [9]). The $R$-matrix (5) can be used to build an endomorphism of the vector space

$$
V^{\otimes \ell} \otimes V^{\otimes \ell}=V_{1} \otimes \ldots \otimes V_{\ell} \otimes V_{\ell+1} \otimes \ldots \otimes V_{2 \ell}
$$

with spectral parameters

$$
\left\{x, q^{2} x, \ldots, q^{2 \ell-2} x, y, q^{2} y, \ldots, q^{2 \ell-2} y\right\}
$$

associated to the $2 \ell$ factors of the tensor product decomposition (14).

Definition 3.1 The operator

$$
R^{(\ell)}(x, y): V^{\otimes \ell} \otimes V^{\otimes \ell} \rightarrow V^{\otimes \ell} \otimes V^{\otimes \ell}
$$

is defined by

$$
\begin{aligned}
R^{(\ell)}(x, y):= & R_{1,2 \ell}\left(x, q^{2 \ell-2} y\right) R_{1,2 \ell-1}\left(x, q^{2 \ell-4} y\right) \cdots R_{1, \ell+1}(x, y) \\
& \times R_{2,2 \ell}\left(q^{2} x, q^{2 \ell-2} y\right) R_{2,2 \ell-1}\left(q^{2} x, q^{2 \ell-4} y\right) \cdots R_{2, \ell+1}\left(q^{2} x, y\right) \\
& \vdots \\
& \times R_{\ell, 2 \ell}\left(q^{2 \ell-2} x, q^{2 \ell-2} y\right) R_{\ell, 2 \ell-1}\left(q^{2 \ell-2} x, q^{2 \ell-4} y\right) \cdots R_{\ell, \ell+1}\left(q^{2 \ell-2} x, y\right) .
\end{aligned}
$$

Given that $R$ satisfies the Yang-Baxter equation Eq. (7) and the inversion relation (8), it can be shown that so does $R^{(\ell)}$.

Proposition 3.2 The matrix $R^{(\ell)}(x, y)$ satisfies the Yang-Baxter equation

$$
R_{23}^{(\ell)}\left(y_{2}, y_{3}\right) R_{13}^{(\ell)}\left(y_{1}, y_{3}\right) R_{12}^{(\ell)}\left(y_{1}, y_{2}\right)=R_{12}^{(\ell)}\left(y_{1}, y_{2}\right) R_{13}^{(\ell)}\left(y_{1}, y_{3}\right) R_{23}^{(\ell)}\left(y_{2}, y_{3}\right)
$$

and the inversion equation

$$
R^{(\ell)}(y, x) R^{(\ell)}(x, y) \propto \mathrm{Id} .
$$


Note that (18) and (19) hold with general spectral parameters; the special choice (15) allows the operator $R^{(\ell)}$ to be restricted to the subspace $\operatorname{Sym}^{\ell} V \otimes \operatorname{Sym}^{\ell} V$, as shown below.

Proposition 3.3 A state $|v\rangle \in V^{\otimes \ell}$ belongs to $\mathrm{Sym}^{\ell} V$ if and only if

$$
R_{i, i+1}\left(q^{2 i-2} x, q^{2 i} x\right)|v\rangle=0
$$

for all $1 \leq i \leq \ell-1$.

Proof It is enough to show that the operator $R_{i, i+1}\left(q^{2 i-2} x, q^{2 i} x\right)$ annihilates a state $|v\rangle$ if and only if $|v\rangle$ is invariant under exchanging positions $i$ and $i+1$, since the $R$-matrix is a local operator. The $R$-matrix for the special choice of spectral parameters $\left(q^{2 i-2} x, q^{2 i} x\right)$ reads as

$$
R_{i, i+1}\left(q^{2 i-2} x, q^{2 i} x\right)=\left(q^{2}-1\right) q^{2 i-2} x\left(\begin{array}{cccc}
0 & 0 & 0 & 0 \\
0 & -1 & 1 & 0 \\
0 & 1 & -1 & 0 \\
0 & 0 & 0 & 0
\end{array}\right)
$$

whose kernel is exactly $\operatorname{Sym}^{2} V \subset V \otimes V$.

Lemma 3.4 The operator $R^{(\ell)}$ leaves $\operatorname{Sym}^{\ell} V \otimes \operatorname{Sym}^{\ell} V$ invariant and therefore the map

$$
R^{(\ell)}(x, y): \operatorname{Sym}^{\ell} V \otimes \operatorname{Sym}^{\ell} V \rightarrow \operatorname{Sym}^{\ell} V \otimes \operatorname{Sym}^{\ell} V
$$

is well-defined.

Proof Let $|v\rangle \otimes|w\rangle \in \mathrm{Sym}^{\ell} V \otimes \operatorname{Sym}^{\ell} V$. Note that the commutation relation

$$
\begin{aligned}
& R_{i, i+1}\left(q^{2 i-2} x, q^{2 i} x\right)\left(R^{(\ell)}(x, y)|v\rangle \otimes|w\rangle\right) \\
& \quad=R^{(\ell)}(x, y)\left(R_{i, i+1}\left(q^{2 i-2} x, q^{2 i} x\right)|v\rangle\right) \otimes|w\rangle
\end{aligned}
$$

holds for $1 \leq i \leq \ell-1$, as a consequence of the Yang-Baxter equation Eq. (7) applied to (17).

Since $|v\rangle$ belongs to $\operatorname{Sym}^{\ell} V$, the r.h.s. of (23) vanishes for $1 \leq i \leq \ell-1$. Therefore, by Proposition 3.3, the vector $R^{(\ell)}(x, y)|v\rangle \otimes|w\rangle$ is symmetric in its first $\ell$ factors. A similar argument shows that $R^{(\ell)}(x, y)|v\rangle \otimes|w\rangle$ is also symmetric in its last $\ell$ factors, and hence $R^{(\ell)}(x, y)|v\rangle \otimes|w\rangle \in \operatorname{Sym}^{\ell} V \otimes \operatorname{Sym}^{\ell} V$.

\subsection{Higher spin model}

Take a $n \times n$ grid as in the 6-vertex model above, and to each edge associate an integer $0 \leq \alpha \leq \ell$, which labels the corresponding state $|\ell ; \alpha\rangle \in \operatorname{Sym}^{\ell} V$. We assume 


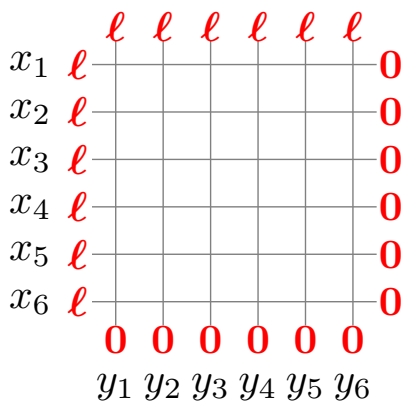

Fig. 3 Boundary conditions and spectral parameters for the $6 \times 6$ grid

generalized domain wall boundary conditions, that is, $\ell$ is assigned to the edges on the left and top boundaries, and 0 is fixed along the edges of the bottom and right boundaries. As in the 6-vertex model, $2 n$ spectral parameters $\{\boldsymbol{x}, \boldsymbol{y}\}$ are associated to the vertical and horizontal lines of the grid (see Fig. 3).

Analogously to the 6-vertex model, the following conservation condition is imposed on a vertex configuration:

$$
\left.\alpha\right|_{\beta} ^{\eta} \gamma \quad \text { such that } \alpha+\beta=\gamma+\eta \text {. }
$$

Using the standard notation

$$
R^{(\ell)}\left(x_{i}, y_{j}\right)|\ell ; \alpha\rangle \otimes|\ell ; \beta\rangle=R_{\alpha, \beta}^{(\ell)} \underset{\alpha, \eta}{\alpha}\left(x_{i}, y_{j}\right)|\ell ; \gamma\rangle \otimes|\ell ; \eta\rangle \text {, }
$$

the weight of a vertex configuration is given by

$$
w_{i, j}\left(x_{i}, y_{j}\right)=R_{\alpha, \beta}^{(\ell)} \underset{\alpha, \eta}{\gamma}\left(x_{i}, q y_{j}\right)
$$

This choice guarantees the integrability of the model.

\subsection{Partition function}

The partition function $Z_{n, \ell}(\boldsymbol{x}, \boldsymbol{y})$ of the spin $\ell$ model is defined exactly as in (2), where now the weights $w_{i j}$ are those given in (25). The fusion process, defining $R^{(\ell)}$ from $R$, allows us to express the partition function of the spin $\ell$ model in terms of the original 6-vertex model partition function as

$$
Z_{n, \ell}(\boldsymbol{x}, \boldsymbol{y})=Z_{\ell n}(\overline{\boldsymbol{x}}, \overline{\boldsymbol{y}}),
$$

where

$$
\overline{\boldsymbol{x}}=\left\{x_{1}, q^{2} x_{1}, \ldots, q^{2 \ell-2} x_{1}, \ldots, x_{n}, q^{2} x_{n}, \ldots, q^{2 \ell-2} x_{n}\right\},
$$


and $\bar{y}$ is defined similarly.

As in (3), the function

$$
\hat{\mathcal{Z}}_{n, \ell}(\boldsymbol{x}, \boldsymbol{y})=\prod_{i}^{n}\left(x_{i} y_{i}\right)^{-\ell / 2} Z_{n, l}(\boldsymbol{x}, \boldsymbol{y})
$$

is a homogeneous polynomial in the variables $x_{i}$ and $y_{i}$. Moreover, $\hat{\mathcal{Z}}_{n, \ell}(\boldsymbol{x}, \boldsymbol{y})$ is divisible by the product

$$
\prod_{i, j}^{n} \prod_{p=0}^{\ell-2} \prod_{k=0}^{\ell-1}\left(q^{2 k} x_{i}-q^{2 p+1} y_{j}\right)
$$

as a consequence of the Izergin-Korepin formula (11) evaluated at $\overline{\boldsymbol{x}}$ and $\overline{\boldsymbol{y}}$. The reduced partition function is defined as

$$
\mathcal{Z}_{n, \ell}(\boldsymbol{x}, \boldsymbol{y}):=\frac{\prod_{i, j=1}^{n} \prod_{p=0}^{\ell} \prod_{k=0}^{\ell-1}\left(q^{2 k} x_{i}-q^{2 p-1} y_{j}\right)}{\Delta(\overline{\boldsymbol{x}}) \Delta(\overline{\boldsymbol{y}})} \operatorname{det} \mathcal{A}_{\ell}(\boldsymbol{x}, \boldsymbol{y}),
$$

where $\mathcal{A}_{\ell}(\boldsymbol{x}, \boldsymbol{y})$ is the $\ell n \times \ell n$ matrix given by

$$
\mathcal{A}_{\ell}(\boldsymbol{x}, \boldsymbol{y}):=\left[A_{\ell}\left(x_{\alpha}, y_{\beta}\right)\right]_{\alpha, \beta=1}^{n}
$$

with $\ell \times \ell$ blocks of the form

$$
A_{\ell}(x, y):=\left[\frac{1}{\left(q^{2 j} x-q^{2 i-1} y\right)\left(q^{2 j} x-q^{2 i+1} y\right)}\right]_{i, j=0}^{\ell-1} .
$$

Note that we recover the Izergin-Korepin determinant when $\ell=1$.

The original partition function can be written as

$$
Z_{n, \ell}(\boldsymbol{x}, \boldsymbol{y})=\text { Const. } \prod_{i}^{n}\left(x_{i} y_{i}\right)^{\ell / 2} \prod_{i, j}^{n} \prod_{p=0}^{\ell-2} \prod_{k=0}^{\ell-1}\left(q^{2 k} x_{i}-q^{2 p+1} y_{j}\right) \mathcal{Z}_{n, \ell}(\boldsymbol{x}, \boldsymbol{y})
$$

where the constant can be determined explicitly.

\section{Alternative representation of the partition function}

For rational functions $u(z)$ and $v(z)$ their residue pairing is defined as

$$
\langle u(z), v(z)\rangle=-\operatorname{Res}_{z=\infty} u(z) v(z)
$$


in terms of which the Izergin-Korepin formula (11) can be presented as

$$
\mathcal{Z}_{n}(\boldsymbol{x}, \boldsymbol{y})=\frac{1}{\Delta(\boldsymbol{x}) \Delta(\boldsymbol{y})} \operatorname{det}_{1 \leq i, j \leq n}\left(\left\langle p_{i}(\boldsymbol{x} ; z), \frac{1}{z-y_{j}}\right\rangle\right),
$$

where

$$
p_{i}(\boldsymbol{x} ; z)=\prod_{\substack{k=1 \\ k \neq i}}^{n}\left(z-q x_{k}\right)\left(z-q^{-1} x_{k}\right), \quad i=1, \ldots, n
$$

In what follows, we use the following alternative representation of the partition function:

Proposition 4.1 The standard 6-vertex model partition function $\mathcal{Z}_{n}(\boldsymbol{x}, \boldsymbol{y})$ can be written as

$$
\mathcal{Z}_{n}(\boldsymbol{x}, \boldsymbol{y})=\operatorname{det}_{1 \leq i, j \leq n}\left(\left\langle r_{i}(\boldsymbol{x} ; z), \frac{z^{j-1}}{w(\boldsymbol{y} ; z)}\right\rangle\right)
$$

where

$$
w(\boldsymbol{y} ; z)=\prod_{k=1}^{n}\left(z-y_{k}\right)
$$

and

$$
r_{i}(\boldsymbol{x} ; z)=\frac{w(\boldsymbol{x} ; q z)\left(q^{-1} z\right)^{i-1}-w\left(\boldsymbol{x} ; q^{-1} z\right)(q z)^{i-1}}{\left(q-q^{-1}\right) z}, \quad i=1, \ldots, n .
$$

Proof First observe that if $\Delta(\boldsymbol{y}) \neq 0$ then

$$
\operatorname{span}_{1 \leq j \leq n}\left\{\frac{1}{z-y_{j}}\right\}=\underset{1 \leq j \leq n}{\operatorname{span}}\left\{\frac{z^{j-1}}{w(\boldsymbol{y} ; z)}\right\}
$$

and the change of basis is given explicitly as

$$
\frac{z^{i-1}}{w(\boldsymbol{y} ; z)}=\sum_{j=1}^{n} M_{i j}(\boldsymbol{y}) \frac{1}{z-y_{j}}, \quad i=1, \ldots, n
$$

where $M$ is the $n \times n$ matrix

$$
M_{i j}(\boldsymbol{y})=\frac{y_{j}^{i-1}}{\prod_{k \neq j}\left(y_{j}-y_{k}\right)}, \quad i, j=1, \ldots n .
$$


Similarly, if $\Delta(\boldsymbol{x}) \neq 0$ then

$$
\underset{1 \leq j \leq n}{\operatorname{span}}\left\{p_{j}(\boldsymbol{x} ; z)\right\}=\underset{1 \leq j \leq n}{\operatorname{span}}\left\{r_{j}(\boldsymbol{x} ; z)\right\}
$$

and the change of basis can be written, using again (42), as

$$
r_{i}(\boldsymbol{x} ; z)=\sum_{j=1}^{n} M_{i j}(\boldsymbol{x}) p_{j}(\boldsymbol{x}, z)
$$

To conclude the proof, it is enough to note that

$$
\operatorname{det}(M(\boldsymbol{x}))=\frac{(-1)^{\left(\begin{array}{c}
n \\
2
\end{array}\right)}}{\Delta(\boldsymbol{x})}
$$

Lemma 4.2 The higher spin partition function is given by

$$
\mathcal{Z}_{n, \ell}(\boldsymbol{x}, \boldsymbol{y})=\operatorname{det}_{1 \leq i, j \leq n \ell}\left(\left\langle\tilde{r}_{i}(\boldsymbol{x} ; z), \frac{z^{j-1}}{w(\overline{\boldsymbol{y}} ; z)}\right\rangle\right)
$$

where

$$
\tilde{r}_{i}(\boldsymbol{x} ; z)=q^{n(\ell-1)} \frac{w(\boldsymbol{x} ; q z)\left(q^{-1} z\right)^{i-1}-w\left(\boldsymbol{x} ; q^{-2 \ell+1} z\right)(q z)^{i-1}}{\left(q-q^{-1}\right) z}
$$

for $1 \leq i \leq n \ell$.

Proof Define the polynomial

$$
\pi(\boldsymbol{x} ; z)=q^{n(\ell-1)^{2}} \prod_{k=1}^{\ell-1} w\left(\boldsymbol{x} ; q^{-2 k+1} z\right)
$$

Observe that

$$
\begin{aligned}
w(\overline{\boldsymbol{x}} ; q z) & =q^{n(\ell-1)} \pi(\boldsymbol{x} ; z) w(\boldsymbol{x} ; q z) \\
w\left(\overline{\boldsymbol{x}} ; q^{-1} z\right) & =q^{n(\ell-1)} \pi(\boldsymbol{x} ; z) w\left(\boldsymbol{x} ; q^{-2 \ell+1} z\right),
\end{aligned}
$$

and hence

$$
r_{i}(\overline{\boldsymbol{x}} ; z)=\pi(\boldsymbol{x} ; z) \tilde{r}_{i}(\boldsymbol{x} ; z), \quad i=1, \ldots, n \ell .
$$


The identity (46) follows by factoring out the product

$$
\prod_{j=1}^{n} \prod_{k=0}^{\ell-1} \pi\left(x ; q^{2 k} y_{j}\right)
$$

of the 1.h.s. of (37).

Proposition 4.3 The partition function $\mathcal{Z}_{n, \ell}(\boldsymbol{x}, \boldsymbol{y})$ satisfies the following properties:

(i) $\mathcal{Z}_{n, \ell}(\boldsymbol{x}, \boldsymbol{y})$ is a homogeneous polynomial in the set of variables $\{\boldsymbol{x}, \boldsymbol{y}\}$,

(ii) $\mathcal{Z}_{n, \ell}(\boldsymbol{x}, \boldsymbol{y})$ is symmetric in the variables $\boldsymbol{x}$ and in the variables $\boldsymbol{y}$,

(iii) $\mathcal{Z}_{n, \ell}(\boldsymbol{x}, \boldsymbol{y})=\mathcal{Z}_{n, \ell}(\boldsymbol{y}, \boldsymbol{x})$,

(iv) $\mathcal{Z}_{n, \ell}(\boldsymbol{x}, \boldsymbol{y})$ has total degree at most $\ell n(n-1)$,

(v) $\mathcal{Z}_{n, \ell}(\boldsymbol{x}, \boldsymbol{y})$ has degree at most $\ell(n-1)$ in each variable $x_{i}$ or $y_{i}$.

Proof The exchange symmetry (iii) follows easily from the representation (30). The determinantal representation (46) shows that $Z_{n}(\boldsymbol{x}, \boldsymbol{y})$ is a symmetric homogeneous polynomial in $\boldsymbol{x}$, and therefore in $\boldsymbol{y}$. The total degree of the partition function can be read off from (30).

To compute the degree of $\mathcal{Z}_{n, \ell}(\boldsymbol{x}, \boldsymbol{y})$ in the variable $y_{1}$, note that (46) can be slightly modified as

$$
\mathcal{Z}_{n, \ell}(\boldsymbol{x}, \boldsymbol{y})=\frac{(-1)^{\left(\begin{array}{c}
n \ell \\
2
\end{array}\right)}}{\Delta(\overline{\boldsymbol{y}})} \operatorname{det}_{1 \leq i, j \leq n \ell}\left(\left\langle\tilde{r}_{i}(\boldsymbol{x} ; z), \frac{1}{z-\bar{y}_{j}}\right\rangle\right)
$$

where only the first $\ell$ columns of the determinant depend on $y_{1}$. The degree of the polynomial $\tilde{r}_{i}(\boldsymbol{x} ; z)$ in $z$ is equal to $n+i-2$, and therefore, the $i$ th row in the first $n \ell \times \ell$ block of the matrix in (53) consists of polynomials of degree $n+i-2$ in the variable $y_{1}$. This means that the highest possible exponent of $y_{1}$ appearing in the determinant (53) is equal to $\sum_{k=1}^{\ell}(n+n \ell-k-1)=n \ell(\ell+1)-\frac{1}{2} \ell(\ell+3)$. To conclude $(v)$, it is enough to recall that the degree of $\Delta(\bar{y})$ in the variable $y_{1}$ is $\frac{1}{2} \ell(\ell-1)+(n-1) \ell^{2}$.

Remark 4.4 In general, the partition function $\mathcal{Z}_{n, \ell}(\boldsymbol{x}, \boldsymbol{y})$ is not fully symmetric in the set of $2 n$ variables $\{\boldsymbol{x}, \boldsymbol{y}\}$.

\section{The combinatorial point}

In this section we present our main result: at the combinatorial point, $q=$ $\exp (2 \pi i /(2 \ell+1))$, the partition function $\mathcal{Z}_{n, \ell}(\boldsymbol{x}, \boldsymbol{y})$ is a certain Macdonald polynomial.

\subsection{The main result}

Let $\ell \delta_{n}$ be the staircase partition with $n$ steps $2 \times \ell$, that is $\ell \delta_{n}=(\ell(n-1), \ell(n-$ $1), \ldots, \ell, \ell, 0,0)$. Notice that the total degree and the degree in each variable $x_{i}$ or $y_{i}$ 
of the partition function $\mathcal{Z}_{n, \ell}(\boldsymbol{x}, \boldsymbol{y})$ are equal to, respectively, $\left|\ell \delta_{n}\right|=\ell n(n-1)$ and the first part of $\ell \delta_{n}$. Let

$$
\rho_{\ell}=e^{2 \pi i /(2 \ell+1)}
$$

and see [16] for the definition of the Macdonald polynomials $P_{\lambda}(x ; q, t)$.

Theorem 5.1 At the combinatorial point $q=\rho_{\ell}$, the partition function $\mathcal{Z}_{n, \ell}(\boldsymbol{x}, \boldsymbol{y})$ is a Macdonald polynomial up to a multiplicative constant, more precisely

$$
\mathcal{Z}_{n, \ell}(\boldsymbol{x}, \boldsymbol{y})=\gamma_{n, \ell} P_{\ell \delta_{n}}\left(\boldsymbol{x}, \boldsymbol{y} ; \rho_{\ell}^{2}, \rho_{\ell}\right) \text {. }
$$

The proportionality constant $\gamma_{n, \ell}$ will be given explicitly in Proposition 5.16.

Remark 5.2 The coefficients $u_{\lambda \mu}(q, t)$ in the expansion of the Macdonald polynomial

$$
P_{\lambda}(z ; q, t)=\sum_{\mu \leq \lambda} u_{\lambda \mu}(q, t) m_{\mu}(z)
$$

are rational functions of $q$ and $t$, and therefore, the specialization $q=\rho_{\ell}^{2}$ and $t=\rho_{\ell}$ has to be done carefully. This issue is addressed in Sect. 5.3.

Theorem 5.1 has the following important consequence:

Corollary 5.3 At the combinatorial point $q=\rho_{\ell}$, the partition function $\mathcal{Z}_{n, \ell}(\boldsymbol{x}, \boldsymbol{y})$ is a fully symmetric polynomial.

In order to prove Theorem 5.1, we show below that the partition function $\mathcal{Z}_{n, \ell}(\boldsymbol{x}, \boldsymbol{y})$ satisfies a set of constraints, known as the wheel condition, see Theorem 5.9. The wheel condition together with the properties described in Proposition 4.3 is sufficient to characterize the partition function up to a multiplicative constant, see Lemma 5.11. Using a result of Feigin et al., we prove that there is a unique Macdonald polynomial of total degree $\ell n(n-1)$ which also satisfies the wheel condition, see Proposition 5.14. Therefore the uniqueness result of Lemma 5.11 implies Theorem 5.1.

5.2 The wheel condition

Let $z=\left\{z_{1}, \ldots, z_{2 n}\right\}$.

Definition 5.4 [Wheel condition] Let $q$ and $t$ be such that $q^{r-1} t^{k+1}=1$, for some non-negative integers $k$ and $r$. A function $f(z)$ is said to obey the $(r, k)_{q, t}$-wheel condition if $f(z)$ vanishes whenever

$$
\frac{z_{i_{\alpha+1}}}{z_{i_{\alpha}}}=t q^{s_{\alpha}} \text { for any } s_{\alpha} \in \mathbb{N} \text { such that } \sum_{\alpha=1}^{k} s_{\alpha} \leq r-1,
$$

and for any choice of $1 \leq i_{1}<i_{2}<\ldots<i_{k+1} \leq 2 n$. 
Lemma 5.5 Let $n \geq 3$. At the combinatorial point $q=\rho_{\ell}$, the partition function $\mathcal{Z}_{n, \ell}(\boldsymbol{x}, \boldsymbol{y})$ vanishes whenever

$$
x_{3}=q^{1+2 s_{2}} x_{2}=q^{2+2 s_{1}+2 s_{2}} x_{1}
$$

for any $s_{1}, s_{2} \in \mathbb{N}$ such that $s_{1}+s_{2} \leq \ell-1$.

Proof Let $\mathcal{S}=\operatorname{span}_{1 \leq i \leq n}\left\{r_{i}(\overline{\boldsymbol{x}} ; z)\right\}$. The polynomial

$$
\begin{aligned}
a(\overline{\boldsymbol{x}} ; z)= & \left(\prod_{i=4}^{n} \prod_{k=0}^{\ell-1}\left(z-q^{2 k} x_{i}\right)\right)\left(\prod_{k=0}^{s_{1}-1}\left(z-q^{2 k} x_{1}\right)\right) \\
& \times\left(\prod_{k=s_{1}}^{s_{1}+s_{2}-1}\left(z-q^{1+2 k} x_{1}\right)\right)\left(\prod_{k=s_{1}+s_{2}}^{\ell-2}\left(z-q^{2+2 k} x_{1}\right)\right)
\end{aligned}
$$

of degree $\ell(n-2)-1$, is such that

$$
\frac{w(\overline{\boldsymbol{x}} ; q z) a\left(\overline{\boldsymbol{x}} ; q^{-1} z\right)-w\left(\overline{\boldsymbol{x}}, q^{-1} z\right) a(\overline{\boldsymbol{x}} ; q z)}{\left(q-q^{-1}\right) z}=0 .
$$

Thus $\operatorname{dim} \mathcal{S}<\ell n$. This implies that the matrix appearing in Eq. (46) is singular.

Lemma 5.6 Let $n \geq 2$. At the combinatorial point $q=\rho_{\ell}$, the partition function $\mathcal{Z}_{n, \ell}(\boldsymbol{x}, \boldsymbol{y})$ vanishes whenever

$$
y_{1}=q^{1+2 s_{2}} x_{2}=q^{2+2 s_{1}+2 s_{2}} x_{1}
$$

for any $s_{1}, s_{2} \in \mathbb{N}$ such that $s_{1}+s_{2} \leq \ell-1$.

Proof Notice that $\bar{y}_{\ell-s_{1}-s_{2}+j}=q^{2 j-1} x_{1}$ for $0 \leq j \leq s_{1}$, because $y_{1}=q^{2+2 s_{1}+2 s_{2}} x_{1}$. Let $\tilde{\mathcal{S}}=\operatorname{span}_{1 \leq i \leq n}\left\{\tilde{r}_{i}(\boldsymbol{x} ; z)\right\}$ and let

$$
a_{i}(x ; z)=z^{i-1} \prod_{j=0}^{s_{1}-1}\left(z-q^{2 j} x\right)
$$

for $1 \leq i \leq \ell n-s_{1}$. Then the polynomials

$$
r_{i}^{\prime}(\overline{\boldsymbol{x}} ; z)=\frac{w(\boldsymbol{x} ; q z) a_{i}\left(x_{1} ; q^{-1} z\right)-w\left(\boldsymbol{x} ; q^{-2 \ell+1} z\right) a_{i}\left(x_{1} ; q z\right)}{\left(q-q^{-1}\right) z}
$$

belonging to $\tilde{\mathcal{S}}$, vanish at $z=q^{2 j-1} x_{1}$ for $0 \leq j \leq s_{1}$. 
Let $A_{i j}=\left\langle\tilde{r}_{i}(\boldsymbol{x} ; z), \frac{1}{z-\bar{y}_{j}}\right\rangle$, then the partition function is given by

$$
\mathcal{Z}_{n, \ell}(\boldsymbol{x}, \boldsymbol{y})=\frac{(-1)^{\left(\begin{array}{c}
\ell n \\
2
\end{array}\right)}}{\Delta(\bar{y})} \operatorname{det}_{1 \leq i, j \leq n \ell}\left(A_{i j}\right)
$$

Let $\mathcal{S}^{\prime}=\operatorname{span}_{1 \leq i \leq \ell n-s_{1}}\left\{r_{i}^{\prime}(\boldsymbol{x} ; z)\right\}$ be a subspace of $\tilde{\mathcal{S}}$, and let $A_{i j}^{\prime}=\left\langle r_{i}^{\prime}(\boldsymbol{x} ; z), \frac{1}{z-\bar{y}_{j}}\right\rangle$. The entries $A_{i j}^{\prime}$ vanish when $\ell-s_{1}-s_{2} \leq j \leq \ell-s_{2}$, so $A^{\prime}$ is of rank at most $\ell n-s_{1}-1$. Therefore $A$ is of rank at most $\ell n-1$.

Lemma 5.7 Let $n \geq 2$. At the combinatorial point $q=\rho_{\ell}$, the partition function $\mathcal{Z}_{n, \ell}(\boldsymbol{x}, \boldsymbol{y})$ vanishes whenever

$$
y_{2}=q^{1+2 s_{2}} y_{1}=q^{2+2 s_{1}+2 s_{2}} x_{1}
$$

for any $s_{1}, s_{2} \in \mathbb{N}$ such that $s_{1}+s_{2} \leq \ell-1$.

Proof This follows from Lemma 5.6, using the symmetries of the partition function. $\square$

Lemma 5.8 Let $n \geq 3$. At the combinatorial point $q=\rho_{\ell}$, the partition function $\mathcal{Z}_{n, \ell}(\boldsymbol{x}, \boldsymbol{y})$ vanishes whenever

$$
y_{3}=q^{1+2 s_{2}} y_{2}=q^{2+2 s_{1}+2 s_{2}} y_{1}
$$

for any $s_{1}, s_{2} \in \mathbb{N}$ such that $s_{1}+s_{2} \leq \ell-1$.

Proof Recall that $\mathcal{Z}_{n, \ell}(\boldsymbol{x}, \boldsymbol{y})=\mathcal{Z}_{n, \ell}(\boldsymbol{y}, \boldsymbol{x})$, and hence this follows from Lemma 5.5.ロ

By summarizing the above, we obtain the following crucial property of the partition function at the combinatorial point:

Theorem 5.9 At the combinatorial point $q=\rho_{\ell}$, the partition function $\mathcal{Z}_{n, \ell}(\boldsymbol{x}, \boldsymbol{y})$ satisfies the $(\ell, 2) \rho_{\ell}^{2}, \rho_{\ell}$-wheel condition.

Definition 5.10 Let $\boldsymbol{x}=\left\{x_{1}, \ldots, x_{n}\right\}$ and $\boldsymbol{y}=\left\{y_{1}, \ldots, y_{n}\right\}$. The vector space $V_{n}$ is defined as the space of polynomials $p(\boldsymbol{x}, \boldsymbol{y})$ such that

(i) $p(\boldsymbol{x}, \boldsymbol{y})$ is a homogeneous polynomial in the set of variables $\{\boldsymbol{x}, \boldsymbol{y}\}$,

(ii) $p(\boldsymbol{x}, \boldsymbol{y})$ is symmetric in the variables $\boldsymbol{x}$, and also in the variables $\boldsymbol{y}$,

(iii) $p(\boldsymbol{x}, \boldsymbol{y})=p(\boldsymbol{y}, \boldsymbol{x})$,

(iv) $p(\boldsymbol{x}, \boldsymbol{y})$ has total degree at most $\ln (n-1)$,

(v) $p(\boldsymbol{x}, \boldsymbol{y})$ has degree at most $\ell(n-1)$ in each variable $x_{i}$ or $y_{i}$,

(vi) $p(\boldsymbol{x}, \boldsymbol{y})$ satisfies the $(\ell, 2) \rho_{\ell}^{2}, \rho_{\ell}$-wheel condition.

Lemma 5.11 The vector space $V_{n}$ is at most one-dimensional.

The proof of the above lemma is given in Appendix 1. 
5.3 The wheel condition and Macdonald polynomials

Definition 5.12 [Admissible partitions] A partition $\lambda=\left(\lambda_{1}, \lambda_{2}, \ldots\right)$ is said to be $(r, k)$-admissible if and only if $\lambda_{i}-\lambda_{i+k} \geq r$ for all $i$.

According to the result of Feigin et al [6], the space of symmetric polynomials satisfying the wheel condition is spanned by Macdonald polynomials. More precisely

Theorem 5.13 ([6]). Let $q$ and $t$ be two generic scalars such that $q^{r-1} t^{k+1}=1$, and let $\mathcal{V}$ denote the space of symmetric polynomials in $z$ satisfying the $(r, k)_{q, t}$-wheel condition. Let $\mathcal{M}$ be the space spanned by the Macdonald polynomials $P_{\lambda}(z ; q, t)$ indexed by $(r, k)$-admissible partitions. Then $\mathcal{V}=\mathcal{M}$.

In the case of relevance for our purposes, this simplifies to

Proposition 5.14 Let $q$ and $t$ be two generic scalars such that $q^{\ell-1} t^{3}=1$, and let $p(z)$ be a homogeneous symmetric polynomial of total degree $\ln (n-1)$ satisfying the $(\ell, 2)_{q, t}$-wheel condition. Then

$$
p(z) \propto P_{\ell \delta_{n}}(z ; q, t) .
$$

Proof The partition $\lambda=\ell \delta_{n}$ is the only $(\ell, 2)$-admissible partition such that $|\lambda|=$ $\ln (n-1)$.

Because we do not use generic $q$ and $t$, extreme care is required before we apply the theorem of Feigin et al. Let $m=\operatorname{gcd}(3, \ell-1)$. The locus of the equation $q^{\ell-1} t^{3}=1$ splits into $m$ branches: $q^{(\ell-1) / m} t^{3 / m}=\omega$, where $\omega$ is a $m$-th root of unity. We consider the branch corresponding to $\omega=\exp (2 \pi i / m)$, parametrized by a variable $u$ :

$$
\begin{array}{lll}
q(u)=u^{3} & t(u)=u^{-(\ell-1)} & \text { for } \ell \equiv 0,2(\bmod 3) \\
q(u)=u & t(u)=u^{-\frac{\ell-1}{3}} e^{\frac{2 \pi i}{3}} & \text { for } \ell \equiv 1(\bmod 3) .
\end{array}
$$

Let $u_{0}$ be such that $q\left(u_{0}\right)=\rho_{\ell}^{2}$ and $t\left(u_{0}\right)=\rho_{\ell}$. Explicitly

$$
\begin{array}{lll}
u_{0}=e^{\frac{4 \pi i}{3(2 \ell+1)}} e^{\frac{2 \pi i}{3}} & \text { for } \ell \equiv 0 & (\bmod 3) \\
u_{0}=e^{\frac{4 \pi i}{2 \ell+1}} & \text { for } \ell \equiv 1 & (\bmod 3) \\
u_{0}=e^{\frac{4 \pi i}{3(2 \ell+1)}} e^{-\frac{2 \pi i}{3}} & \text { for } \ell \equiv 2 & (\bmod 3) .
\end{array}
$$

In [6], it was shown that the coefficients $u_{\lambda \mu}(q(u), t(u))$ in the expansion (56) are well-defined rational functions in $u$. When $u$ approaches $u_{0}$, the coefficients $u_{\ell \delta_{n}, \mu}(q(u), t(u))$ behave like $\left(u-u_{0}\right)^{n_{\mu}}$ for some power $n_{\mu}$. Let $N=-\min _{\mu}\left\{n_{\mu}\right\}$, which is non-negative because $n_{\ell \delta_{n}}=0$. Then the renormalized polynomial

$$
\tilde{P}_{\ell \delta_{n}}(z ; q(u), t(u))=\left(u-u_{0}\right)^{N} P_{\ell \delta_{n}}(z ; q(u), t(u))
$$

is a regular function when $u$ approaches $u_{0}$. 
Notice that $\tilde{P}_{\ell \delta_{n}}(z ; q(u), t(u))$ is a homogeneous symmetric polynomial of total degree $\ln (n-1)$ satisfying the wheel condition when $u \neq u_{0}$. The limit $\lim _{u \rightarrow u_{0}} \tilde{P}_{\ell \delta_{n}}(z ; q(u), t(u))$ is well-defined, and it is simply given by

$$
\lim _{u \rightarrow u_{0}} \tilde{P}_{\ell \delta_{n}}(z ; q(u), t(u))=\tilde{P}_{\ell \delta_{n}}\left(z ; \rho_{\ell}^{2}, \rho_{\ell}\right)
$$

Lemma 5.15 The polynomial $\tilde{P}_{\ell \delta_{n}}\left(z ; \rho_{\ell}^{2}, \rho_{\ell}\right)$ satisfies the $(\ell, 2) \rho_{\ell}^{2}, \rho_{\ell}$-wheel condition.

Proof This lemma follows from the regularity of $\tilde{P}_{\ell \delta_{n}}(z ; q(u), t(u))$ when $u$ approaches $u_{0}$.

5.4 The proof of the main theorem

Let

$$
[\ell]_{q} !=[\ell]_{q}[\ell-1]_{q} \ldots[1]_{q}, \text { where }[a]_{q}=\frac{q^{a}-1}{q-1}
$$

Proposition 5.16 The constant $\gamma_{n, \ell}$, defined in Theorem 5.1, is given by

$$
\gamma_{n, \ell}=\left((-1)^{\left(\begin{array}{c}
\ell \\
2
\end{array}\right)} \rho_{\ell}^{2\left(\begin{array}{l}
\ell \\
2
\end{array}\right)(2 n-3)}[\ell] \rho_{\ell}^{2 !}\right)^{n} .
$$

In order to prove this proposition, in Appendix 2 we compute the coefficient of $\prod_{i=1}^{n}\left(x_{i} y_{i}\right)^{\ell(i-1)}$ in the partition function. Since this coefficient is non-zero, the Macdonald polynomial $P_{\ell \delta_{n}}\left(\boldsymbol{x}, \boldsymbol{y} ; \rho_{\ell}^{2}, \rho_{\ell}\right)$ is well-defined and

$$
P_{\ell \delta_{n}}\left(\boldsymbol{x}, \boldsymbol{y} ; \rho_{\ell}^{2}, \rho_{\ell}\right)=\tilde{P}_{\ell \delta_{n}}\left(\boldsymbol{x}, \boldsymbol{y} ; \rho_{\ell}^{2}, \rho_{\ell}\right) .
$$

Proof (Proof of Theorem 5.1) Note that the definition of $V_{n}$ is such that $\mathcal{Z}_{n, \ell}(\boldsymbol{x}, \boldsymbol{y}) \in$ $V_{n}$, and $P_{\ell \delta_{n}}\left(\boldsymbol{x}, \boldsymbol{y} ; \rho_{\ell}^{2}, \rho_{\ell}\right) \in V_{n}$. But by Lemma 5.11, $V_{n}$ is at most one-dimensional, and therefore, $\mathcal{Z}_{n, \ell}(\boldsymbol{x}, \boldsymbol{y})=\gamma_{n, \ell} P_{\ell \delta_{n}}\left(\boldsymbol{x}, \boldsymbol{y} ; \rho_{\ell}^{2}, \rho_{\ell}\right)$, with coefficient $\gamma_{n, \ell}$ computed in Proposition 5.16.

\section{Final remarks}

\subsection{Combinatorial interpretation}

A canonical higher spin generalization of an alternating sign matrix (ASM), as defined by Behrend and Knight [1], is a square matrix with entries in $\{-\ell, \ldots,-1,0,1, \ldots, \ell\}$, such that sum of all entries in each row or column is $\ell$, and the partial sum of the first or last $r$ entries in each row or column is non-negative for each $r$. The bijection between 
such matrices and the configurations of the model considered in this paper can be established similarly to the case $\ell=1$ : reading each column of the grid from the bottom to the top, if the value of the edge goes from $\beta$ to $\eta$, the corresponding entry of the higher spin ASM is $\eta-\beta$. Alternatively, the conservation condition $\alpha+\beta=\gamma+\eta$ guarantees that the same result is obtained by reading each row from right to left. Unfortunately, the weights considered in this paper seem very unnatural in the combinatorial setting.

By setting all variables of the partition function $\mathcal{Z}_{n}(\boldsymbol{x}, \boldsymbol{y})$ to be equal to 1 at the combinatorial point $q=\exp (2 \pi i / 3)$, we get the famous sequence

$$
1,1,2,7,42,429,7436,218348, \ldots \quad \text { (sequence A005130 in OEIS) }
$$

that counts several objects, including alternating sign matrices and totally symmetric self-complementary plane partitions. We have seen that at the combinatorial point $q=\exp ((2 \ell+1) / 2 \pi i)$, the higher spin partition function $\mathcal{Z}_{n, \ell}(\boldsymbol{x}, \boldsymbol{y})$ is a Macdonald polynomial. Therefore, it would be interesting to find some combinatorial interpretation of the values obtained for $\ell>1$ in the homogeneous limit when $x_{i}=y_{i}=1$ for all $i$.

\subsection{The wheel condition}

The wheel condition for the higher spin partition function is deduced using the special determinantal form $\mathcal{Z}_{n, \ell}(\boldsymbol{x}, \boldsymbol{y})$ that involves rational function entries with a specific dependence on the crossing parameter $q$. This motivates a more systematical study of the connection between the wheel condition and more general determinants of a similar type. See, for example [15], for a different generalization of the IzerginKorepin determinant that satisfies a wheel condition.

There are other integrable statistical models that are of interest from this point of view, such as, for example, the $\mathfrak{s o}_{n}$ model in [5] and the eight-vertex model in [22,25].

\subsection{Symmetry}

It is very intriguing that we start with a function that exhibits an $S_{n} \times S_{n}$ symmetry but not $S_{2 n}$ symmetry in general. It is natural to ask what the special features of this determinant are that imply the extra symmetry at $q=\exp ((2 \ell+1) / 2 \pi i)$. It would be expected that the symmetry comes from the physics of the model, that is, there exists some mechanism (like the Yang-Baxter equation) which, for this very special value of $q$, allows us to exchange a row with a column.

\subsection{Relation to KP $\tau$-functions}

The alternative representation (46) of the partition function has a Grassmannian manifold interpretation, and this point of view is intimately connected with the fact that the 6-vertex model with domain wall boundary conditions can be seen as a $\tau$-function of the KP hierarchy, where the spectral parameters $\boldsymbol{x}$ and $\boldsymbol{y}$ play the role of Miwa variables associated to the commuting flows of the hierarchy. This was first observed 
by Foda et al. [7], based on a fermionic vacuum expectation value representation of the partition function. In his survey paper [24], Takasaki extends this and other related results by showing how partition functions of certain 2D solvable models and scalar products of Bethe vectors from integrable spin chain models can also be written as $\mathrm{KP} \tau$-functions.

Acknowledgments The authors thank the Centre de Recherches Mathématiques in Montréal where most of the research presented here was carried out. The work of T. F. was partially supported by ANR project DIADEMS (Developing an Integrable Approach to Dynamical and Elliptic Models). The work of F. B. was partially supported by the FP7 IRSES project RIMMP (Random and Integrable models in Mathematical Physics), the ERC project FroM-PDE (Frobenius Manifolds and Hamiltonian Partial Differential Equations) and the MIUR Research Project Geometric and analytic theory of Hamiltonian systems in finite and infinite dimensions. The authors are grateful to the referees for their constructive remarks to improve the structure and the readability of the paper.

\section{Appendix 1: Uniqueness}

The goal of this section is to prove Lemma 5.11. That is, if there exists a non-zero polynomial $p(\boldsymbol{x}, \boldsymbol{y}) \in V_{n}$, then it is unique up to a multiplicative constant.

Let $z=\left\{z_{1}, \ldots, z_{2 n}\right\}=\{\boldsymbol{x}, \boldsymbol{y}\}$ and $q=\rho_{\ell}$. We generalize the $(\ell, 2)_{q^{2}, q^{\text {-wheel }}}$ condition:

Definition 6.1 ( $r$-wheel condition). Let $r$ be such that $3 r<\ell$. A polynomial $p(z)$ is said to obey the $r$-wheel condition if it vanishes whenever

$$
z_{k}=q^{1+2 r+2 s_{2}} z_{j}=q^{2+4 r+2 s_{1}+2 s_{2}} z_{i}
$$

for any $s_{1}, s_{2} \in \mathbb{N}$ such that $s_{1}+s_{2} \leq \ell-1-3 r$ and any choice of $1 \leq i<j<k \leq 2 n$.

The following lemma holds:

Lemma 6.2 Let $a(\boldsymbol{x}, \boldsymbol{y}) \in V_{n}$ be such that $\left.a(\boldsymbol{x}, \boldsymbol{y})\right|_{y_{j}=q x_{i}}=0$ for a given $i$ and $j$. Then $a(\boldsymbol{x}, \boldsymbol{y})=0$.

Proof The result is trivial when $n=1$. Assume $n>1$. By symmetry, $a(\boldsymbol{x}, \boldsymbol{y}) \propto$ $\prod_{i, j}\left(y_{j}-q x_{i}\right)\left(x_{i}-q y_{j}\right)$, therefore, there exists a polynomial $b(\boldsymbol{x}, \boldsymbol{y})$ such that

$$
a(\boldsymbol{x}, \boldsymbol{y})=\left(\prod_{i, j}\left(y_{j}-q x_{i}\right)\left(x_{i}-q y_{j}\right)\right) b(\boldsymbol{x}, \boldsymbol{y}) .
$$

The polynomial $b(\boldsymbol{x}, \boldsymbol{y})$ has total degree at most $(\ell-2) n(n-1)-2 n$ and degree at most $(\ell-2)(n-1)-2$ in each variable $x_{i}$ or $y_{i}$. When $\ell \leq 2, b(\boldsymbol{x}, \boldsymbol{y})=0$ and the lemma is proved.

By the wheel condition, the following holds:

$$
\left.b(\boldsymbol{x}, \boldsymbol{y})\right|_{x_{j}=q x_{i}}=\left(\prod_{k \neq i, j} \prod_{s=1}^{\ell}\left(x_{k}-q^{2 s} x_{i}\right)\right)\left(\prod_{k} \prod_{s=2}^{\ell-1}\left(y_{k}-q^{2 s} x_{i}\right)\right) b^{\prime}(\boldsymbol{x}, \boldsymbol{y}) .
$$


The polynomial $b^{\prime}(\boldsymbol{x}, \boldsymbol{y})$ has degree at most $-2 n$ in $x_{i}$, and therefore it vanishes identically. Thus,

$$
a(\boldsymbol{x}, \boldsymbol{y})=w_{1}(\boldsymbol{x}, \boldsymbol{y}) a_{1}(\boldsymbol{x}, \boldsymbol{y}),
$$

for some polynomial $a_{1}(\boldsymbol{x}, \boldsymbol{y})$ obeying the 1-wheel condition, and

$$
w_{\alpha}(z)=\prod_{i \neq j}\left(z_{i}-q^{\alpha} z_{j}\right)
$$

The polynomial $a_{1}(z)$ has total degree at most $(\ell-4) n(n-1)-2 n$, and it has degree at most $(\ell-4)(n-1)-2$ in each variable $x_{i}$ or $y_{i}$.

By the 1-wheel condition, the following holds:

$$
\left.a_{1}(z)\right|_{z_{j}=q^{3} z_{i}}=\left(\prod_{k \neq i, j} \prod_{s=0}^{\ell-4}\left(z_{k}-q^{6+2 s} z_{i}\right)\right) a_{1}^{\prime}(z) .
$$

The polynomial $a_{1}^{\prime}(z)$ has degree at most $-2(n-1)-4$ in $z_{i}$, and therefore it vanishes identically. Thus,

$$
a_{1}(z)=w_{3}(z) a_{2}(z)
$$

This procedure can be iterated. In the $r$-th step, we define $a_{r}(z)$ by

$$
a_{r-1}(z)=w_{2 r-1}(z) a_{r}(z)
$$

The polynomial $a_{r}(z)$ obeys the $r$-wheel condition, has total degree at most $(\ell-$ $4 r) n(n-1)-2 r n$ and has degree at most $(\ell-4 r)(n-1)-2 r$ in each variable $z_{i}$. The $r$-wheel condition implies

$$
\left.a_{r}(\boldsymbol{z})\right|_{z_{j}=q^{1+2 r} z_{i}}=\left(\prod_{k \neq i, j} \prod_{s=0}^{\ell-1-3 r}\left(z_{k}-q^{2+4 r+2 s} z_{i}\right)\right) a_{r}^{\prime}(\boldsymbol{z})
$$

The polynomial $a_{r}^{\prime}(z)$ viewed as a function of $z_{i}$ has degree at most $-2 r(n-1)-4 r$, and therefore, it vanishes identically. This closes the iteration step.

We should stop the iteration at $r^{*}=\min \{r \in \mathbb{N}$ such that $3 r \geq \ell\}$. The polynomial $a_{r^{*}}(z)$ has negative total degree, and therefore, it vanishes identically.

Proof (Proof of Lemma 5.11) Use induction on $n$. Let $p_{n}(\boldsymbol{x}, \boldsymbol{y}) \in V_{n}$.

The lemma holds for $n=1$. By the wheel condition the following holds:

$$
\left.p_{n}(\boldsymbol{x}, \boldsymbol{y})\right|_{y_{j}=q x_{i}}=\left(\prod_{k \neq i} \prod_{s=1}^{\ell}\left(x_{k}-q^{2 s} x_{i}\right)\right)\left(\prod_{k \neq j} \prod_{s=1}^{\ell}\left(y_{k}-q^{2 s} x_{i}\right)\right) \hat{p}_{n}(\boldsymbol{x}, \boldsymbol{y}) .
$$


The polynomial $\hat{p}_{n}(\boldsymbol{x}, \boldsymbol{y})$ does not depend either on $x_{i}$ or on $y_{j}$. It can then be checked that $\hat{p}_{n}(z) \in V_{n-1}$, which by the induction hypothesis is one-dimensional.

For any non-zero polynomial $p_{n}^{\prime}(\boldsymbol{x}, \boldsymbol{y}) \in V_{n}$, there is a constant $\alpha$ such that

$$
\left.\left(p_{n}(\boldsymbol{x}, \boldsymbol{y})-\alpha p_{n}^{\prime}(\boldsymbol{x}, \boldsymbol{y})\right)\right|_{y_{j}=q x_{i}}=0
$$

By application of Lemma 6.2 the proof is complete.

\section{Appendix 2: The coefficient $\gamma_{n, \ell}$}

\section{Proposition 6.3 The recursion}

$$
\left.\mathcal{Z}_{n, \ell}(\boldsymbol{x}, \boldsymbol{y})\right|_{x_{n}=y_{n}=0}=(-1)^{\left(\begin{array}{c}
\ell \\
2
\end{array}\right)} q^{4\left(\begin{array}{c}
\ell \\
2
\end{array}\right)(n-1)-\left(\begin{array}{c}
\ell \\
2
\end{array}\right)}[\ell]_{q^{2}} !\left(\prod_{k=1}^{n-1} x_{k}^{\ell} y_{k}^{\ell}\right) \mathcal{Z}_{n-1, \ell}(\hat{\boldsymbol{x}}, \hat{\boldsymbol{y}})
$$

holds, where $\hat{\boldsymbol{x}}=\left(x_{1}, \ldots, x_{n-1}\right)$ and $\hat{\boldsymbol{y}}=\left(y_{1}, \ldots, y_{n-1}\right)$.

Proof The determinant representation (46) of $\mathcal{Z}_{n, \ell}(\boldsymbol{x}, \boldsymbol{y})$ implies that

$$
\left.\mathcal{Z}_{n, \ell}(\boldsymbol{x}, \boldsymbol{y})\right|_{x_{n}=y_{n}=0}=\operatorname{det}_{1 \leq i, j \leq n \ell}\left(\left\langle\left.\tilde{r}_{i}(\boldsymbol{x} ; z)\right|_{x_{n}=0}, \frac{z^{j-1}}{w(\overline{\hat{\boldsymbol{y}}} ; z) z^{\ell}}\right\rangle\right) .
$$

Observe that

$$
\left.\tilde{\boldsymbol{r}}_{\ell+i}(\boldsymbol{x} ; z)\right|_{x_{n}=0}=z^{\ell+1} \tilde{\boldsymbol{r}}_{i}(\hat{\boldsymbol{x}} ; z) \quad i \geq 1
$$

Note also, assuming $y_{i} \neq 0$ for $1 \leq i \leq n-1$, that

$$
\operatorname{span}_{1 \leq j \leq n \ell}\left\{\frac{z^{j-1}}{w(\overline{\hat{y}} ; z) z^{\ell}}\right\}=\operatorname{span}\left(\left\{\frac{1}{z^{\ell-j+1}}\right\}_{j=1}^{\ell} \cup\left\{\frac{z^{j-1}}{w(\boldsymbol{y} ; z)}\right\}_{j=1}^{(n-1) \ell}\right),
$$

with the change of basis

$$
\frac{z^{i-1}}{w(\hat{\hat{y}} ; z) z^{\ell}}=\sum_{j=1}^{\ell} M_{i, j}(\hat{\boldsymbol{y}}) \frac{1}{z^{\ell-j+1}}+\sum_{j=1}^{(n-1) \ell} M_{i, \ell+j}(\hat{\boldsymbol{y}}) \frac{z^{j-1}}{w(\overline{\hat{y}} ; z)}
$$

where the matrix $M(\hat{\boldsymbol{y}})$ defined by (90) is upper triangular, and its determinant is equal to

$$
\operatorname{det}(M(\hat{\boldsymbol{y}}))=\frac{1}{w(\overline{\hat{\boldsymbol{y}}} ; 0)^{\ell}}=(-1)^{(n-1) \ell} \frac{q^{-(n-1) \ell^{2}(\ell-1)}}{\prod_{k=1}^{n-1} y_{k}^{\ell^{2}}}
$$


A simple calculation gives that

$$
\begin{aligned}
& \left\langle\left.\tilde{r}_{i}(\boldsymbol{x} ; z)\right|_{x_{n}=0}, \frac{1}{z^{\ell-j+1}}\right\rangle \\
& \quad= \begin{cases}0 & \text { if } i>\ell-j+1 \\
(-1)^{n-1} q^{n(\ell-1)+i-2 \ell+1}[\ell-i+1]_{q^{2}} \prod_{k=1}^{n-1} x_{k} & \text { if } i=\ell-j+1 .\end{cases}
\end{aligned}
$$

By combining the above, we obtain

$$
\begin{aligned}
\left.\mathcal{Z}_{n, \ell}(\boldsymbol{x}, \boldsymbol{y})\right|_{x_{n}=y_{n}=0}= & \operatorname{det}(M(\hat{\boldsymbol{y}})) \operatorname{det}_{1 \leq i, j \leq \ell}\left(\prod_{j=1}^{n}\left\langle\left.\tilde{r}_{i}(\boldsymbol{x} ; z)\right|_{x_{n}=0}, \frac{1}{z^{\ell-j+1}}\right\rangle\right) \\
& \times \operatorname{det}_{1 \leq i, j \leq(n-1) \ell}\left(\left\langle z^{\ell+1} \tilde{r}_{i}(\hat{\boldsymbol{x}} ; z), \frac{z^{j-1}}{w(\hat{\hat{\boldsymbol{y}}} ; z)}\right\rangle\right) .
\end{aligned}
$$

A short calculation gives

$$
\begin{aligned}
& \operatorname{det}_{1 \leq i, j \leq \ell}\left(\prod_{j=1}^{n}\left\langle\left.\tilde{r}_{i}(\boldsymbol{x} ; z)\right|_{x_{n}=0}, \frac{1}{z^{\ell-j+1}}\right\rangle\right) \\
& =(-1)^{\left(\begin{array}{l}
\ell \\
2
\end{array}\right)+(n-1) \ell} q^{n(\ell-1) \ell-\frac{3 \ell(\ell-1)}{2}}[\ell]_{q^{2}} !\left(\prod_{k=1}^{n-1} x_{k}\right)^{\ell},
\end{aligned}
$$

and we also have

$$
\begin{aligned}
& \operatorname{det}_{1 \leq i, j \leq(n-1) \ell}\left(\left\langle z^{\ell+1} \tilde{r}_{i}(\hat{\boldsymbol{x}} ; z), \frac{z^{j-1}}{w(\hat{\hat{\boldsymbol{y}}} ; z)}\right\rangle\right) \\
& =q^{(\ell-1) \ell(\ell+1)(n-1)}\left(\prod_{k=1}^{n-1} y_{k}^{\ell(\ell+1)}\right) \mathcal{Z}_{n-1, \ell}(\hat{\boldsymbol{x}}, \hat{\boldsymbol{y}}),
\end{aligned}
$$

from which the recursion formula (86) follows.

Proof (Proof of Prop. 5.16) Note that

$$
\tilde{r}_{i}(x ; z)=q^{i-\ell}[\ell-i+1]_{q^{2}} z^{i-1}+\text { lower order terms in } z,
$$

and hence

$$
\begin{aligned}
\mathcal{Z}_{1, \ell}(x, y) & =q^{-\left(\begin{array}{l}
\ell \\
2
\end{array}\right)}[\ell]_{q^{2}} ! \operatorname{det}\left(\left\langle z^{i-1}, \frac{z^{j-1}}{w(\bar{y} ; z)}\right\rangle\right)_{i, j=1}^{n \ell} \\
& =(-1)^{\left(\begin{array}{l}
\ell \\
2
\end{array}\right)} q^{-\left(\begin{array}{l}
\ell \\
2
\end{array}\right)[\ell]_{q^{2}} !} .
\end{aligned}
$$


The relation (86) can be used recursively to compute the coefficient of the leading term $\prod_{i=1}^{n}\left(x_{i} y_{i}\right)^{\ell(i-1)}$ in $\mathcal{Z}_{n, \ell}(\boldsymbol{x}, \boldsymbol{y})$ which gives

$$
\left((-1)^{\left(\begin{array}{l}
\ell \\
2
\end{array}\right)} q^{\left(\begin{array}{l}
\ell \\
2
\end{array}\right)(2 n-3)}[\ell]_{q^{2}} !\right)^{n},
$$

that coincides with the proportionality constant $\gamma_{n, \ell}$ since, by definition, the Macdonald polynomial $P_{\ell \delta_{n}}(\boldsymbol{x}, \boldsymbol{y} ; q, t)$ has leading coefficient 1 .

\section{References}

1. Behrend, R.E., Knight, V.A.: Higher spin alternating sign matrices. Electron. J. Combin. 14, 38 (2007). Research Paper 83, http://www.combinatorics.org/ojs/index.php/eljc/article/view/v14i1r83

2. Bressoud, D.: Proofs and Confirmations: The Story of the Alternating Sign Matrix Conjecture. MAA Spectrum Mathematical Association of America, Washington, DC (1999)

3. Caradoc, A., Foda, O., Kitanine, N.: Higher spin vertex models with domain wall boundary conditions. J. Stat. Mech. 2006(03), P03012 (2006). doi:10.1088/1742-5468/2006/03/P03012

4. de Gier, J.: Loops, matchings and alternating-sign matrices. Discrete Math 298(1-3), 365-388 (2005). doi:10.1016/j.disc.2003.11.060

5. Dow, A., Foda, O.: On the domain wall partition functions of level-1 affine so(n) vertex models. J. Stat. Mech. 2006(05), P05010 (2006). doi:10.1088/1742-5468/2006/05/P05010

6. Feigin, B., Jimbo, M., Miwa, T., Mukhin, E.: Symmetric polynomials vanishing on the shifted diagonals and Macdonald polynomials. Int. Math. Res. Not. 2003(18), 1015-1034 (2003)

7. Foda, O., Wheeler, M., Zuparic, M.: Domain wall partition functions and KP. J. Stat. Mech. 2009(3), P03017 (2009). doi:10.1088/1742-5468/2009/03/P03017

8. Fonseca, T.: Alternating sign matrices, completely packed loops and plane partitions, Ph.D. thesis, Université Pierre et Marie Curie, (2010). http://tel.archives-ouvertes.fr/tel-00521884/fr/

9. Fulton, W., Harris, J.: Representation Theory, Graduate Texts in Mathematics. A First Course, Readings in Mathematics, vol. 129. Springer, New York (1991)

10. Izergin, A.G.: Partition function of a six-vertex model in a finite volume. Dokl. Akad. Nauk SSSR 297(2), 331-333 (1987)

11. Korepin, V.: Calculation of norms of Bethe wave functions. Comm. Math. Phys. 86(3), 391-418 (1982). http://www.springerlink.com/content/x6j8w6j351673132

12. Kulish, P., Reshetikhin, N., Sklyanin, E.: Yang-Baxter equation and representation theory: I. Lett. Math. Phys. 5, 393-403 (1981)

13. Kuperberg, G.: Another proof of the alternating-sign matrix conjecture, Internat. Math. Res. Not. 3, 139-150 (1996). doi:10.1155/S1073792896000128

14. Kuperberg, G.: Symmetry classes of alternating-sign matrices under one roof. Ann. of Math. 3, 835-866 (2002). doi:10.2307/3597283

15. Lascoux, A.: Gaudin functions, and Euler-Poincaré characteristics (2007). arXiv:0709.1635

16. Macdonald, I.G.: Symmetric functions and Hall polynomials. Oxford Mathematical Monographs. Oxford University Press Inc., Oxford (1979)

17. Mills, W., Robbins, D., Rumsey Jr, H.: Alternating sign matrices and descending plane partitions. J. Combin. Theory Ser. A 34(3), 340-359 (1983)

18. Mills, W., Robbins, D., Rumsey Jr, H.: Self-complementary totally symmetric plane partitions. J. Combin. Theory Ser. A 42(2), 277-292 (1986)

19. Okada, S.: Enumeration of symmetry classes of alternating sign matrices and characters of classical groups. J. Algebraic Combin. 23(1), 43-69 (2006)

20. Pimenta, R.A., Martins, M.J.: The Yang-Baxter equation for PT invariant 19-vertex models. J. Phys. A 44(8), 085205 (2011). arXiv: 1010.1274

21. Reshetikhin, N.: Lectures on the Integrability of the 6-Vertex Model, Exact Methods in Lowdimensional Statistical Physics and Quantum Computing, Lecture Notes of the Les Houches Summer School, vol. 89 (2010). arXiv:1010.5031 
22. Rosengren, H.: An Izergin-Korepin-type identity for the 8VSOS model, with applications to alternating sign matrices. Adv. Appl. Math. 43(2), 137-155 (2009)

23. Stroganov, Yu G.: Izergin-Korepin determinant at a third root of unity. Theoret. Math. Phys. 146(1), 53-62 (2006)

24. Takasaki, K.: KP and Toda tau functions in Bethe Ansatz, New Trends in Quantum Integrable Systems, pp. 373-391, World Scientific Publishing, Hackensack (2011). arXiv:1003.3071

25. Yang, W.-L., Zhang, Y.-Z.: Partition function of the eight-vertex model with domain wall boundary condition. J. Math. Phys. 50(8), 083518,14 (2009) 